\title{
Prevalencia de disfunción eréctil y factores asociados en pacientes con indicación de rehabilitación cardíaca
}

\author{
Claudio Santibáñez, Claudia Anchique, Artur Herdy, Cecilia Zeballos, Graciela González, Rosalía Fernández, Ma. Virginia \\ Araya, Paula Soto. \\ 1. Departamento de Prevención y Rehabilitación, Centro Cardiológico S\&S, Puerto Montt, Chile. \\ 2. División de Enf. Cardiovasculares, Rehabilitación Cardiaca, Mediagnóstica, Duitama, Colombia. \\ 3. Instituto de Cardiología de Santa Catarina, Brasil. \\ 4. Servicio de Rehabilitación Cardíaca, Instituto Cardiovascular de Buenos Aires, Argentina. \\ 5. Programa de Prevención y Rehabilitación Cardiovascular, H. Central Instituto de Previsión Social, Asunción, Paraguay. \\ 6. Unidad de Rehabilitación Cardíaca, Instituto Nacional Cardiovascular "Carlos A. Pashchiera", Lima, Perú. \\ 7. Red de Prevención Cardiovascular del Norte Grande, Centro médico, Iquique, Chile. \\ a. Directora y Docente Escuela Enfermería Universidad Austral de Chile, Puerto Montt. \\ Grupo Sudamericano de Prevención Y Rehabilitación Cardiaca
}

Introducción: La disfunción eréctil (DE) afecta a millones de personas en el mundo. Se caracteriza por daño endotelial vascular y actualmente es considerado un marcador de riesgo de enfermedad cardiovascular. Sin embargo, no es evaluada normalmente por el personal médico y los pacientes se rehúsan a discutirlo. El objetivo de este trabajo es determinar la prevalencia de DE y sus factores asociados en pacientes con indicación de rehabilitación cardíaca.

Material y Método: Estudio descriptivo, transversal, incluyó 225 pacientes con indicación de rehabilitación cardíaca de 6 países de Latinoamérica: Chile $(\mathrm{CH})$, Brasil (BR), Perú (PE), Colombia (CO), Argentina (AR) y Paraguay (PY) en el período de marzo-agosto 2014. Se obtuvo datos demográficos y se utilizó el cuestiona- rio Índice Internacional de Función Eréctil (CIIFE) auto administrado. Las variables fueron procedencia, edad, peso, talla, IMC, obesidad, hipertensión arterial (HTA), diabetes mellitus (DM), infarto agudo del miocardio (IAM), tabaquismo y uso de fármacos.

Resultados: Se evaluó a 225 hombres. De acuerdo al CIIFE, 80,1\% tuvo algún tipo de DE; 8,9\% severa; $10,7 \%$ moderada; $26,2 \%$ media a moderada y $35,6 \%$ media. Los factores asociados significativamente fueron el país de procedencia, la DM, la edad y el tabaquismo. Conclusión: La prevalencia de Disfunción Eréctil en pacientes con indicación de rehabilitación cardíaca de Chile, Brasil, Perú, Colombia, Argentina y Paraguay fue $80,1 \%$ y sus factores asociados son edad, obesidad, IAM, DM, HTA y tabaquismo.

\section{Correspondencia:}

Dr. Claudio Santibáñez Catalán.

Dirección: Benavente 840, Edificio Baquedano oficina 701,

Puerto Montt, Chile. Celular 98837927.

csanti4@yahoo.com 


\section{Erectile dysfunction in patients referred for cardiac rehabilitation: prevalence and associated factors}

Background: Erectile dysfunction (ED) affects millions of males around the world. It is characterized by endothelial dysfunction and is currently considered to be a marker of cardiovascular disease. However, it is seldom evaluated by medical personnel and patients frequently refuse to discuss the subject. The aim of this investigation was to determine incidence and prevalence of ED and associated factors in patients referred for cardiac rehabilitation.

Method: A descriptive, cross sectional study was performed in 225 patients referred for cardiac rehabilitation in 6 Latin American countries: Chile $(\mathrm{CH})$, Brazil (BR), Perú (PE), Colombia (CO), Argentina (AR) and Paraguay (PY), from March to August 2014. Demographic data were collected and the self-administered International ED questionnaire was applied to evaluate
ED. Variables included age, weight, height, BMI, obesity, hypertension (HT), diabetes mellitus (DM), acute myocardial infarction, smoking habit and use of different medications

Results: $80.1 \%$ of subjects had some type of ED: it was severe in $8.9 \%$, moderate in $10.7 \%$. moderate to intermediate in $26.2 \%$ and intermediate in $35.6 \%$. Factors associated to the presence of $\mathrm{ED}$ were country of origin, DM, age, and smoking habit

Conclusion: ED was present in a mean of $80.1 \%$ of patients referred for cardiac rehabilitation in Chile, Brazil, Perú, Colombia, Argentina, and Paraguay. ED was associated to age, obesity, myocardial infarction, DM, HT, and smoking habit

Kewwords: erectile dysfunction; rehabilitation, cardiac; Latin America 


\section{Introducción}

La disfunción eréctil (DE), definida por el National Institute of Health (NIH)como "la incapacidad de alcanzar o mantener la erección del pene lo suficiente para lograr una relación sexual satisfactoria" tiene dos causas principales: orgánica (vascular, traumática / post quirúrgica, neurológicas, endocrinas e inducidas por fármacos) y psicógena (depresión, ansiedad, y sociabilización) ${ }^{2}$. Corresponde a un problema de alta incidencia mundial que se estima en 152 millones en 1995 y se pronostica que ascenderá a 322 millones en el año $2025^{1}$.

La DE es frecuente en pacientes con patología cardiovascular. En múltiples estudios se ha demostrado que la DE y la ECV son diferentes manifestaciones de una enfermedad vascular común. Desafortunadamente, aún existe un sub registro de los pacientes con DE por parte de los trabajadores de salud e incluso reparo de los pacientes a consultar oportunamente su problema sexual. Es por esto que se decidió medir el grado de función eréctil utilizando el cuestionario Índice Internacional de Función Eréctil (IIEF) en 225 pacientes de RCV de 6 países de Latinoamérica, validado en 12 países y diez idiomas, incluido el español. ${ }^{33}$

Se han desarrollado muchos perfiles de función sexual masculina y cuestionarios de DE. El IIEF es la medida más fiable de $\mathrm{DE}^{3}$. Cuenta con 15 preguntas y la puntuación total se obtiene mediante la suma de las puntuaciones individuales de cada pregunta. Aborda y cuantifica cinco ámbitos, a saber: la función de erección, la función orgásmica (eyaculación), el deseo sexual (libido), satisfacción de las relaciones sexuales (capacidad de mantener relaciones sexuales), y la satisfacción / eyaculación precoz en general. ${ }^{32,33,34}$

Este estudio tuvo como objetivo determinar la prevalencia de la disfunción eréctil en los candidatos a un programa de rehabilitación cardíaca, determinar la gravedad de la disfunción eréctil y describir los factores que se asocian a esta condición.

\section{Material y métodos}

Estudio transversal, analítico y descriptivo. Se realizó muestreo por conglomerado y conveniencia. Durante el período de marzo a agosto 2014. Se incluyeron 225 pacientes masculinos con indicación de rehabilitación cardíaca (RHC) de 6 países de Latinoamérica: 23,1\% Chile (Ch), 24\% Brasil (BR), 13,8\% Perú (PE), 13,3\% Colombia (CO), 13,3\% Argentina (AR), 12,4\% Paraguay (PY). Previa firma de consentimiento informado, se obtuvieron sus datos demográficos y se utilizó el Índice In- ternacional de Función Eréctil (IIEF), cuestionario auto administrado que consta de 5 preguntas con 5 opciones de respuesta cada una, permitiendo clasificar la DE en severa (puntuación 5-7), moderada (8-11), media a moderada (12-16), media (17-21) y no sufre DE (22-25). Las variables estudiadas fueron procedencia, edad, peso, talla, IMC, Fracción Eyección, obesidad, hipertensión arterial (HTA), diabetes mellitus (DM), infarto agudo del miocardio (IAM), tabaquismo, uso de hipolipemiantes, betabloqueadores, diuréticos, IECA, ARAII, anticoagulante oral, hipoglucemiantes, antidepresivos. Se utilizó estadística descriptiva y analítica y para asociación chi2 y Pearson con software SPSS (V22).

\section{Resultados}

Se evaluaron 225 pacientes masculinos de 6 centros de rehabilitación cardíaca en Latinoamérica, obteniendo un $55,6 \%$ mayores de 60 años, peso 79,8 $\pm 11,9 \mathrm{~kg}$, estatura $1,68 \pm 0,07 \mathrm{mts}, 25,8 \%$ obesos, $72,4 \%$ HTA, $39,6 \%$ diabetes mellitus (DM), 63,1\% IAM previo y $20 \%$ con historia de tabaquismo. De acuerdo al IIEF, $80,1 \%$ tuvo algún tipo de DE; 8,9\% severa, 10,7\% moderada, 26,2\% media a Moderada y 35,6\% media.

La prevalencia de DE por cada país fue: $72,2 \% \mathrm{BR}$, $76,7 \%$ AR, $80,8 \%$ h, 83,9\% PE, 89,3\% PY y $90 \%$ CO, El mayor promedio de DE Severa fue Ch con $21,2 \%$, en Moderada y Media a Moderada fue CO con $20 \%$ y $40 \%$, respectivamente, y en Media fue PE con 54,8\%.

Los factores asociados significativamente a la disfunción eréctil fueron el país de procedencia, la DM, la edad y el tabaquismo. En los pacientes con DE Severa se encontró asociación significativa sólo con la procedencia, edad, uso de ARAII y antecedente de IAM. En DE moderada sólo la DM, en DE media a moderada el Tabaquismo y uso de ARAII, y DE media sólo la procedencia.

\section{Discusión}

Existen algunos estudios basados en población Latinoamericana en relación a la prevalencia y correlación de la DE en diversos escenarios culturales y étnicos. Actualmente, según el conocimiento disponible, este es el primer estudio basado en población en seis países Latinoamericanos para reportar la prevalencia de DE en pacientes con indicación de rehabilitación cardíaca. Diversos estudios han evaluado la prevalencia de la DE en Brasil 12-15, 18, 24, 27, 31, Argentina 20, 21 y Colombia ${ }^{18,23}$, más no en Chile, Perú ni Paraguay.

Existe una alta prevalencia de DE en la muestra de estudio, atribuible a la relación entre DE y riesgo de acciden- 
te cardiovascular, comparable con numerosos estudios tanto europeos $6,9,10,11,25,26,30$, como Latinoamericanos ${ }^{17-19,22,23,27}$. No así estudios en que se ha documentado la prevalencia de DE de forma aislada en amplios universos muestrales, incluyendo pacientes masculinos de toda índole y edad, obteniendo niveles de prevalencia relativamente bajos como es el caso del estudio EDEM en España, realizado a 2476 pacientes de 25-70 años, donde diagnosticaron DE con prevalencia de $18,9 \%$ con IIEF. En la mayoría de otros estudios sí se han hallado valores de prevalencia en DE leve, moderados a altos en un intervalo entre $17.2 \%$ a 49,9\% (Italia, Malasia Austria, Francia, Japón y Jordania) ${ }^{11,} 30$ en Europa y Asia, como también en Latinoamérica: Un estudio transversal (2006) en Sao Paulo Brasil de 2,862 hombres arrojó una prevalencia de $45.1 \%{ }^{15}$; Otro estudio en 2001 en Buenos Aires, Argentina de 2715 hombres obtuvo una prevalencia de DE de $41.7 \%{ }^{20}$. El primer estudio realizado en el norte de Sudamérica en 2002 DENSA de 1946 hombres, obtuvo valores de prevalencia mínimos, moderados y completos o severos para DE de $53.4 \%$ (Colombia, $52.8 \%$; Ecuador, 52.1\%; Venezuela, 55.2\%), siendo la prevalencia de DE mínima, moderada y severa similares a los hallados en los diferentes países del mundo ${ }^{33}$. Sin embargo, se desconocen en la actualidad estudios para Chile, Perú y Paraguay.

Por numerosos estudios aplicados a diversas muestras demográficas a nivel mundial es altamente reconocida la relación existente entre la DE y la ECV, eventos arteriales periféricos, y coronarios, tanto en Europa, Asia y Oceanía, como también en países americanos. En Italia se estudiaron 300 pacientes con patología coronaria comprobada encontrando una prevalencia de DE de 49\%. Se determinó, además, el intervalo de tiempo entre la DE y la aparición de ECV, la cual fue de 38,8 meses. ${ }^{9}$ En Austria, de 2495 pacientes de los cuales 163 presentaban DE moderada/severa (prevalencia del 6,5\%) poseían un $65 \%$ más de riesgo para desarrollar ECV en los próximos 10 años ${ }^{11}$. Brant et al, ${ }^{10}$ realizaron un seguimiento a 1402 pacientes durante 10 años, donde determinaron la asociación entre la prevalencia de DE y la incidencia de ECV, concluyendo que cuando la DE ocurre en hombres jóvenes está asociada con un marcado aumento en el riesgo de un futuro evento cardíaco. En 2003 Montorsi et $\mathrm{al}^{9}$ evaluaron a 300 pacientes con dolor precordial y enfermedad coronaria, encontrando una prevalencia de DE de $49 \%$, donde además lograron evidenciar que los síntomas de DE precedieron a la angina en un $67 \%$ de ellos. En Viena Ponholzer et $\mathrm{al}^{11}$, después de estudiar a un grupo de 2495 hombres sin ECV, concluyeron que la DE moderada a severa está asociada con un considerable aumento del riesgo de padecer una ECV en los próximos 10 años (2005). Hodges et al, en 2007 compararon un grupo de 207 pacientes con ECV y 165 sin ECV, encontrando una incidencia de DE de $66 \%$ y $43 \%$ respectivamente $(\mathrm{p}<0,05)$, concluyendo que la DE podría preceder un evento cardiovascular aproximadamente en 5 años ${ }^{6}$. Jia-Yi Dong et al, 2011 China, en un meta-análisis de 12 estudios de cohorte prospectivos incluyendo 36,744 participantes evidencia que la DE está significativa e independientemente relacionada con el aumento del riesgo de ECV (48\%), enfermedades cardíacas coronarias (46\%), derrame cerebral $(35 \%)$ y mortalidad general $(19 \%)^{30}$. Realidades comparables se han observado en Latinoamérica. En Sao Paulo, Brasil 2010, de 222 pacientes, aquellos con DE presentaron un riesgo cardiovascular mayor según los criterios del Puntaje de Riesgo Framingham (FRS) y las mediciones de Proteína C- reactiva ${ }^{17}$. En Santa Fe y Entre Ríos, Argentina, la prevalencia de enfermedades cardiovasculares relacionadas a la DE fue de $16 \%$, entre otros factores de riesgo (DM, HTA, drogas terapéuticas $)^{19}$. En Buenos Aires, Argentina 2010, a 78 hombres se aplicó el IIEF, cuyos resultados sugirieron que la DE puede considerarse un marcador de daño metabólico y vascular, el cual evolucionaría potencialmente en ECV y DM $2^{22}$. Fafiolu S. et. al en 2014 en Nigeria, de un total de 202 hombres determinó aquellos con edades entre 46 y 64 años tuvieron 3 veces más probabilidad de tener DE con respecto a aquellos menores de 45 años. Además, encontraron una prevalencia del $75 \%$ en hombres con $\mathrm{HTA}^{28}$.

En la presente investigación, los factores asociados significativamente a la disfunción eréctil fueron: el país de procedencia, la edad, DM y el tabaquismo respectivamente, en orden decreciente. Todas estas son condiciones responsables de efectos perjudiciales sobre el endotelio vascular y nervios periféricos y de inducción de daño ultraestructural al tejido corporal, motivos que en conjunto generan $\mathrm{DE}^{29}$. Datos consistentes se han encontrado en 2002 en países al norte de Sudamérica, donde la edad tuvo una fuerte correlación con la DE, seguido por DM e HTA y otros factores de riesgo prominentes ${ }^{18}$. Similares observaciones provienen del estudio DENSA $2002^{23}$, así como de numerosos estudios mundiales 12, 13, 18, 19, 23-29. En los pacientes con DE Severa de este estudio se encontró asociación significativa sólo con la procedencia, edad, uso de ARAII y antecedente de IAM. En DE moderada sólo la DM. En DE media a moderada, el Tabaquismo y 
uso de ARAII, y en DE media sólo la procedencia. Otros factores de riesgo han sido ampliamente descritos en la literatura, como son obesidad, sedentarismo, consumo de alcohol, depresión, uso de drogas terapéuticas, varios de los cuales no fueron abordados en este estudio o no arrojaron correlación estadísticamente significativa con la prevalencia de DE.

\section{Conclusión}

La DE es considerada un problema de salud pública mayor, que afecta seriamente la calidad de vida de los pacientes $^{29}$, suele estar relacionada a importantes co-morbilidades como ECV, DM e HTA, que actúan, en ocasiones, exacerbándola.

Basados en los hallazgos presentados en esta investigación y la literatura consultada puede concluirse que, tal como en otras poblaciones masculinas, la DE es un problema común entre hombres Latinoamericanos, con una prevalencia moderada que aumenta significativamente con la edad, y que es bastante alta en pacientes con antecedentes de ECV e indicación de rehabilitación cardíaca.

\section{Agradecimientos}

Un merecido reconocimiento a cada una de las personas que pertenecen a las unidades de Rehabilitación cardiaca de su respectivo país.

\section{Referencias}

1. NIH CONSENSUS CONFERENCE. Impotence: NIH Consensus Development Panel on Impotence. JAMA. 1993; 270:83-90.

2. NEELIMA V CHU, EDELMAN SV. Diabetes and Erectile Dysfunction. Clinical diabetes. 2001; 19:45-47.

3. KUBIN M, WAGNER G, FUGL-MEYER AR. Epidemiology of erectile dysfunction. Int J Impot Res. 2003; 15:63-71.

4. SHIRI R, HAKAMA M, HÄKKINEN J, TAMMELA TL, AUVINEN A, KOSKIMÄKI J. Relationship between smoking and erectile dysfunction. Int J Impot Res. 2005; 17: 164-9.

5. FELDMAN HA, GOLDSTEIN I, HATZICHRISTOU DG, KRANE RJ, MCKINLAY JB. Impotence and its medical and psychological correlates: results of the Massachusetts Male Ageing Study. J urol. 1994; 151: 54-61.

6. SIU SC, LO SK, WONG KW, IP KM, WONG YS. Prevalence of and risk factors for erectile dysfunction in Hong Kong diabetic patients. Diabet Med. 2001; 18: 732-738.

7. MARUMO K, NAKASHIMA J, MURAI M. Age-related prevalence of erectile dysfunction in Japan:assessment by the International Index of Erectile Function. IntJUrol. 2001; 8: 53-5.
8. NICOLOSI A, MOREIRA ED JR, SHIRAI M, BIN MOHD TAMBI MI, GLASSER DB. Epidemiology of erectile dysfunction in four countries: Cross-national study of the prevalence and correlates of erectile dysfunction. Urology. 2003; 61: 201-6.

9. MUELEMAN EJ. Prevalence of erectile dysfunction: need for treatment? Int J Impot Res. 2002; 14: S22-28.

10. INMAN BA, SAUVER JL, JACOBSON DJ, MCGREE ME, NEHRA A, LIEBER MM, et. al ; A Population-Based, Longitudinal Study of Erectile Dysfunction and Future Coronary Artery Disease. Mayo Clin Proc. 2009; 84: 108-113.

11. PONHOLZER A, TEMML C, OBERMAYR R, WEHRBERGER C, MADERSBACHER S. Is erectile dysfunction an indicator for increased risk of coronary heart disease and stroke? Eur Urol. 2005; 48: 512-8.

12. MOREIRA ED JR, BESTANE WJ, BARTOLO EB, FITTIPALDI JA. Prevalence and determinants of erectile dysfunction in Santos, southeastern Brazil. Sao Paulo Med J. 2002 Mar 7; 120: 49-54.

13. RHODEN EL, TELÖKEN C, SOGARI PR, VARGAS SOUTO CA. The use of the simplified International Index of Erectile 
Function (IIEF-5) as a diagnostic tool to study the prevalence of erectile dysfunction. Int J Impot Res. 2002; 14: 245-50.

14. MOREIRA ED JR, LISBOA LÔBO CF, VILLA M, NICOLOSI A, GLASSER DB. Prevalence and correlates of erectile dysfunction in Salvador, northeastern Brazil: a population-based study. Int J Impot Res. 2002; 14: S3-9.

15. ABDO CH, OLIVEIRA WM JR, SCANAVINO MDE T, MARTINS FG. [Erectile dysfunction: results of the Brazilian Sexual Life Study]. Rev Assoc Med Bras. 2006; 52:424-9.

16. SPESSOTO LC, CORDEIRO JA, DE GODOY JM. Effect of systemic arterial pressure on erectile dysfunction in the initial stages of chronic arterial insufficiency. BJU Int. 2010; 106:1723-5.

17. ZAMBON JP, MENDONÇA RR, WROCLAWSKI ML, KARAM JUNIOR A, SANTOS RD, et al, Cardiovascular and metabolic syndrome risk among men with and without erectile dysfunction: case-control study. Sao Paulo Med J. 2010; 128: $137-40$.

18. BECHER E, GLINA S. Erectile dysfunction in Latin America and treatment with sildenafil citrate (Viagra): introduction. Int J Impot Res. 2002; 14: S1-2.

19. OLIVA A, GIAMI A, MULTIGNER L. Environmental agents and erectile dysfunction: a study in a consulting population. $\mathrm{J}$ Androl. 2002; 23: 546-50.

20. NOLAZCO C, BELLORA O, LÓPEZ M, SURUR D, VÁZQUEZ J, ROSENFELD C, et al. Prevalence of sexual dysfunctions in Argentina. Int J Impot Res. 2004; 16: 69-72.

21. MURAD BAŞAR, TUĞLU D, YILMAZ E, BAŞAR H, BATISLAM E. Relationship between nocturnal penile tumescence parameters, International Index of Erectile Function symptom scores and sildenafil responses. Scand J Urol Nephrol. 2006; 40: 506-10.

22. KNOBLOVITS P, COSTANZO PR, VALZACCHI GJ, GUEGLIO G, LAYUS AO, KOZAK AE, et al. Erectile dysfunction, obesity, insulin resistance, and their relationship with testosterone levels in eugonadal patients in an andrology clinic setting. J Androl. 2010; 31: 263-70.

23. MORILLO LE, DÍAZ J, ESTEVEZ E, COSTA A, MÉNDEZ $\mathrm{H}$, DÁVILA H, et al. Prevalence of erectile dysfunction in Colombia, Ecuador, and Venezuela: a population-based study (DENSA). Int J Impot Res. 2002; 14: S10-8.
24. COSTA MR, REIS AM, PEREIRA BP, PONCIANO VC, OLIVEIRA EC. Associated factors and prevalence of erectile dysfunction in hemodialysis patients. Int Braz J Urol. 2014; 40: 44-55.

25. ALBERTI L, TORLASCO C, LAURETTA L, LOFFI M, MARANTA F, SALONIAA, et al. Erectile dysfunction in heart failure patients: a critical reappraisal. Andrology. 2013; 1: 177-91.

26. VLACHOPOULOS C, JACKSON G, STEFANADIS C, MONTORSI P. Erectile dysfunction in the cardiovascular patient. Eur Heart J. 2013; 34: 2034-46.

27. GONZÁLES AI, STIES SW, WITTKOPF PG, MARA LS, ULBRICH AZ, CARDOSO FL, et al. Validation of the International Index of Erectile Function (IIFE) for use in Brazil. Arq Bras Cardiol. 2013; 101: 176-82.

28. FAFIOLU AS, ADEBAYO AM, AKANDE TO, AKINBOBOYE OO. Erectile dysfunction among male hypertensives in a tertiary health facility in South-West Nigeria. Glob J Health Sci. 2014 Aug 22; 7 : 154-60.

29. TOSTES RC, CARNEIRO FS, LEE AJ, GIACHINI FR, LEITE R, OSAWA Y, et al. Cigarette smoking and erectile dysfunction: focus on NO bioavailability and ROS generation. J Sex Med. 2008; 5: 1284-95.

30. DONG JY, ZHANG YH, QIN LQ. Erectile dysfunction and risk of cardiovascular disease: meta-analysis of prospective cohort studies. J Am Coll Cardiol. 2011 Sep 20; 58: 1378-85.

31. NICOLOSI A, MOREIRA E, SHIRAI M, et. al. Epidemiology of erectile dysfunction in four countries: cross-national study of the prevalence and correlates of erectile dysfunction. Urology. 2003; 61: 201-6.

32. ROSEN RC, CAPPELLERI JC, SMITH MD, LIPSKY J, PEÑA BM. Development and evaluation of the International Index of Erectile Function (IIEF-5) as a diagnostic tool for erectile dysfunction. Int J Imp Res 1999; 11: 319-326.

33. ROSEN RC, RILEY A, WAGNER G, OSTERLOH IH, KIRKPATRICK J, MISHRA A. The International Index of Erectile Function (IIEF): a multidimensional scale for assesment of erectile dysfunction. Urology 1997; 49: 822-830.

34. GARCÍA P, CASTRO J, YUDES E. Prevalencia de la disfunción eréctil en un programa de rehabilitación cardíaca. Enferm Cardiol. 2007; 16: 53-59. 